\title{
ANTI-MAGIC SQUARES OF EVEN ORDER
}

\author{
SHENG JIANG
}

\begin{abstract}
A systematic method for constructing anti-magic squares of every even order $(>2)$ was found. This partially answered an open question asked by Gakuho Abe.
\end{abstract}

\section{Introduction}

An anti-magic square of order $n$ is an $n$ by $n$ matrix with entries $1,2, \ldots, n^{2}$ such that the set of all the sums of $n$ numbers in each row, each column and each main diagonal consists of consecutive numbers.

Gakuho Abe [1] collected several unsolved problems on magic squares, in which the last one is the following

Problem 2.23. Find a method of constructing an anti-magic square of every order.

The aim of this paper is to solve above problem for the case of even orders.

In section 2, we shall prove a classification theorem. It says that all antimagic squares can be divided into two classes, called type + and type - , for each class the consecutive sums are completely determined by order $n$.

Then in section 3 , we consider the case $n=4 k(k=1,2,3, \ldots)$, and give a method of constructing anti-magic squares for these $n$.

Finally, section 4 deals with the same topic for $n=4 k+2(k=1,2,3, \ldots)$, and obtain similar results. And hence Abe's problem 2.23 is solved for all even orders.

\section{Classification}

Let $A=\left(a_{i j}\right)$ be an anti-magic square of order $n$. Denote by $r_{i}$ the sum of $n$ numbers in $i$-th row of $A, c_{j}$ the sum of $j$-th column, $d_{1}$ and $d_{2}$ the sums for each of main diagonals $(i, j=1,2, \ldots, n)$, respectively. Let $S_{0}$ be the average of all $r_{i}(i=1,2, \ldots, n)$, then it is also the average of all $c_{j}(j=1,2, \ldots, n)$, and

$$
S_{0}=\frac{1}{2} n\left(n^{2}+1\right) \text {. }
$$

Received February 2, 2000; revised August 3, 2001. 
Now we can prove the following

THeORem 1. Let $A=\left(a_{i j}\right)$ be an anti-magic square of order $n$. Then in the $(2 n+2)$ sums $d_{1}, d_{2}, r_{i}$ and $c_{j}(i, j=1,2, \ldots, n)$, there is always a set of $(2 n+1)$ sums consists of $\left\{S_{0}, S_{0} \pm 1, \ldots, S_{0} \pm n\right\}$, and the rest one is equal to either $S_{0}-(n+1)$ or $S_{0}+(n+1)$.

Proof. Denote by

$$
s=\frac{1}{2 n+2}\left(d_{1}+d_{2}+\sum_{i=1}^{n} r_{i}+\sum_{j=1}^{n} c_{j}\right),
$$

then

$$
d_{1}+d_{2}=2(n+1) s-n^{2}\left(n^{2}+1\right) .
$$

Let $\alpha$ and $\omega$ be the smallest one and largest one of $(2 n+2)$ sums $d_{1}, d_{2}, r_{i}$ and $c_{j}(i, j=1,2, \ldots, n)$, respectively, then

$$
2 s=\alpha+\omega=2 \alpha+2 n+1 .
$$

Since

we have

$$
2 \alpha+1 \leq d_{1}+d_{2} \leq 2 \omega-1,
$$

$$
\frac{1}{2} n\left(n^{2}-1\right)-\frac{3}{2} \leq \alpha \leq \frac{1}{2} n\left(n^{2}-1\right)+\frac{1}{2} .
$$

But $\alpha$ is an integer, hence only two values are possible:

$$
\alpha_{1}=\frac{1}{2} n\left(n^{2}-1\right)-1, \quad \alpha_{2}=\frac{1}{2} n\left(n^{2}-1\right) .
$$

If $\alpha=\alpha_{1}$, then the $(2 n+2)$ sums are $S_{0}, S_{0} \pm 1, \ldots, S_{0} \pm n$, and $S_{0}-(n+1)$. If $\alpha=\alpha_{2}$, then the $(2 n+2)$ sums are $S_{0}, S_{0} \pm 1, \ldots, S_{0} \pm n$, and $S_{0}+(n+1)$.

Remark. According to Theorem 1, all anti-magic squares can be divided into two classes. For one class, each square has a sum $S_{0}-(n+1)$, and will be called of type - And the other class will be called of type + , in which every square has a sum $S_{0}+(n+1)$.

The Theorem 1 is true for all possible orders. But in the following sections we shall see that even orders maybe are more interesting, because one can get a simple method to construct them.

\section{Order $n=4 k$}

Obviously, any square of order 2 cannot be anti-magic. Hence, for an antimagic square of even order $n$, the smallest possible value of $n$ is 4 .

In this section we consider the case $n=4 k(k=1,2,3, \ldots)$.

Lemma 1. There exist anti-magic squares of order 4 both for type - and type + . 
Proof. Let $P$ be a $4 \times 4$ matrix with entries $1,2,3, \ldots, 16$, such that

$$
P=\left(\begin{array}{ll}
A & B \\
C & D
\end{array}\right)
$$

where $A=\left(a_{\alpha \beta}\right), \quad B=\left(b_{\alpha \beta}\right), \quad C=\left(c_{\alpha \beta}\right)$ and $D=\left(d_{\alpha \beta}\right)$ are $2 \times 2$ submatrices satisfying

$$
a_{\alpha 1}+a_{\alpha 2}=d_{1 \beta}+d_{2 \beta}=b_{\alpha \beta}+c_{\beta \alpha}=17 \quad(\alpha, \beta=1,2) .
$$

By computer search, we get many examples of anti-magic squares in this special form, one is showed in Table 1. It is of type - and denoted by $P^{-}$. The numbers out of frame show the sums of rows, columns and main diagonals of this square.

\begin{tabular}{|c|c|c|c|}
\hline 4 & 13 & 12 & 1 \\
\hline 11 & 6 & 2 & 14 \\
\hline 5 & 15 & 10 & 8 \\
\hline 16 & 3 & 7 & 9 \\
\hline 36 & 37 & 31 & 32 \\
\hline
\end{tabular}

Table 1. $P^{-}$

Further, in the matrix $P^{-}$, changing rows of block $A$ as well as columns of block $D$, we get an anti-magic square of type + as Table 2. Denote the new square by $P^{+}$.

\begin{tabular}{|c|c|c|c|}
\hline 11 & 6 & 12 & 1 \\
\hline 4 & 13 & 2 & 14 \\
\hline 5 & 15 & 8 & 10 \\
\hline 16 & 3 & 9 & 7 \\
\hline
\end{tabular}

Table 2. $P^{+}$

Remark. Squares $P^{-}$and $P^{+}$have the same corresponding row sums $r_{i}$ and column sums $c_{j}$ (for each $i$ and each $j$ ), as well as one diagonal sum $d_{1}$, but only different in the other's. Such a closely related pair will be called twin anti-magic squares.

THEOREM 2. For $n=4 k(k=1,2,3, \ldots)$, there exist anti-magic squares of order $n$ both for type - and type + .

Proof. When $k=1$, i.e. $n=4$, we have squares $P^{-}$and $P^{+}$by Lemma 1 . When $k>1$, we shall construct by bordered block matrix. For convenience, let us consider the case $n=8$ in detail. The procedure contains three steps. 
(1) Substituting $(1 / 2)\left((n-4)^{2}+1\right)=17 / 2$ from every entries of $P^{-}$, we obtain a new square, denoted by $P_{*}^{-}$, as Table 3 .

Table 3. $P_{*}^{-}$

\begin{tabular}{|c|c|c|c|}
\hline-4.5 & 4.5 & 3.5 & -7.5 \\
\hline 2.5 & -2.5 & -6.5 & 5.5 \\
\hline-3.5 & 6.5 & 1.5 & -0.5 \\
\hline 7.5 & -5.5 & -1.5 & 0.5 \\
\hline 2 & 3 & -3 & -2 \\
\hline
\end{tabular}

(2) Now construct an $8 \times 8$ block matrix $M_{8}$ in the form

$$
M_{8}=\left(\begin{array}{ccc}
A^{*} & E & B^{*} \\
F & P_{*}^{-} & G \\
C^{*} & H & D^{*}
\end{array}\right),
$$

where $A^{*}=\left(a_{\alpha \beta}^{*}\right), B^{*}=\left(b_{\alpha \beta}^{*}\right), C^{*}=\left(c_{\alpha \beta}^{*}\right)$ and $D^{*}=\left(d_{\alpha \beta}^{*}\right)$ are $2 \times 2$ submatrices satisfying

$$
a_{\alpha 1}^{*}=-a_{\alpha 2}^{*}, \quad d_{1 \beta}^{*}=-d_{2 \beta}^{*}, \quad b_{\alpha \beta}^{*}=-c_{\beta \alpha}^{*} \quad(\alpha, \beta=1,2) ;
$$

$E=\left(e_{\alpha x}\right), H=\left(h_{\alpha x}\right),{ }^{t} F=\left(f_{\alpha x}\right)$ and ${ }^{t} G=\left(g_{\alpha x}\right)$ (here " $t$ " denotes the transpose of matrix) are $2 \times 4$ submatrices satisfying

$$
\begin{gathered}
e_{1 x}=-e_{2 x}, \quad f_{1 x}=-f_{2 x}, \quad g_{1 x}=-g_{2 x}, \quad h_{1 x}=-h_{2 x} \\
(x=1,2,3,4) .
\end{gathered}
$$

Note that the numbers in blocks $E, F, G$ and $H$ are in the range -31.5 to -8.5 and 8.5 to 31.5 .

Now let us set

$$
\begin{aligned}
\frac{n^{2}-1}{2} & =31.5=c_{21}^{*}=c_{12}^{*}+1=a_{12}^{*}+2 \\
& =a_{21}^{*}+3=d_{11}^{*}+4=d_{22}^{*}+5,
\end{aligned}
$$

and

$$
\begin{aligned}
& b_{11}^{*}=c_{21}^{*}-n=23.5, \\
& b_{22}^{*}=b_{11}^{*}+2=25.5 .
\end{aligned}
$$

For the arragement of numbers in $E, H$ and ${ }^{t} F$, the only requirement is that every sum of 4 numbers in any row equals zero, but the sums for ${ }^{t} G$ are \pm 1 . Thus $M_{8}$ is determined.

(3) Adding to each entries of $M_{8}$ by $\left(n^{2}+1\right) / 2=32.5$, we get an anti-magic square of order 8 as Table 4 , which is of type - and denoted by $M_{8}^{-}$. 
Table 4. $M_{8}^{-}$

\begin{tabular}{|c|c|c|c|c|c|c|c|}
\hline 3 & 62 & 49 & 15 & 14 & 52 & 56 & 1 \\
\hline 61 & 4 & 16 & 50 & 51 & 13 & 2 & 58 \\
\hline 21 & 44 & 28 & 37 & 36 & 25 & 57 & 8 \\
\hline 43 & 22 & 35 & 30 & 26 & 38 & 10 & 55 \\
\hline 42 & 23 & 29 & 39 & 34 & 32 & 11 & 54 \\
\hline 24 & 41 & 40 & 27 & 31 & 33 & 53 & 12 \\
\hline 9 & 63 & 45 & 19 & 18 & 48 & 60 & 6 \\
\hline 64 & 7 & 20 & 46 & 47 & 17 & 5 & 59 \\
\hline 267 & 266 & 262 & 263 & 257 & 258 & 254 & 253 \\
\hline
\end{tabular}

Table 5. $M_{8}^{+}$

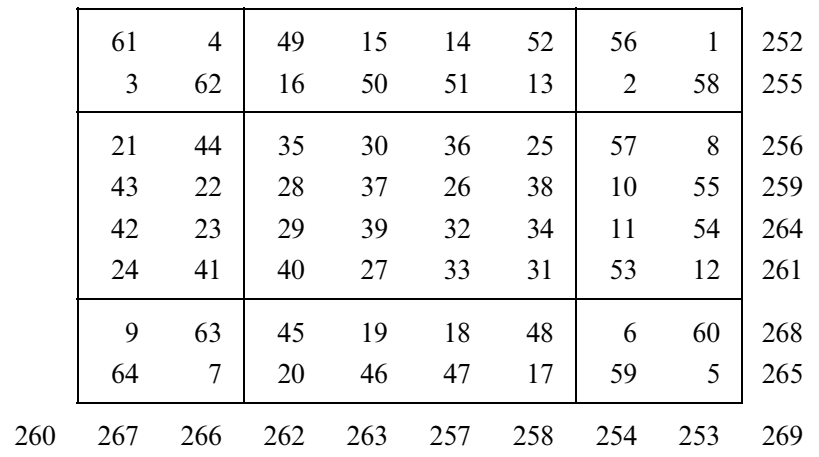

Changing rows or columns in suitable blocks of $M_{8}^{-}$, we obtain an antimagic square of type + , denoted by $M_{8}^{+}$, as Table 5 .

In a similar manner, for any $n=4 k(k=2,3, \ldots)$, we can generate a pair of twin anti-magic squares $M_{n}^{-}$and $M_{n}^{+}$from $M_{n-4}^{-}$or $M_{n-4}^{+}$.

\section{Order $n=4 k+2$}

The rest even orders we need consider are of form $n=4 k+2$ $(k=1,2,3, \ldots)$.

Lemma 2. There exist anti-magic squares of order 6 both for type - and type + .

Proof. Using block matrix and by means of computer search, we get a pair of twin anti-magic squares of order 6 . They are denoted by $Q^{-}$and $Q^{+}$, and showed in Tables 6 and 7, respectively. 
Table 6. $Q^{-}$

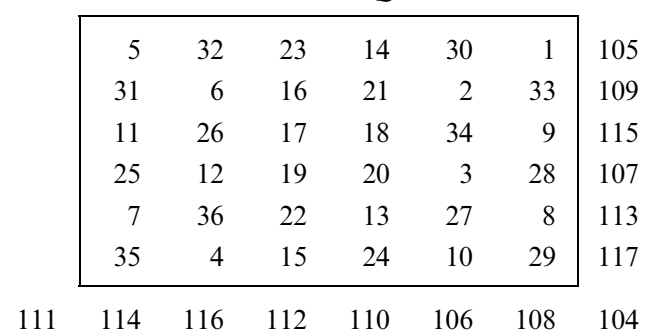

Table 7. $Q^{+}$

\begin{tabular}{|c|c|c|c|c|c|}
\hline 31 & 6 & 23 & 14 & 30 & 1 \\
\hline 5 & 32 & 16 & 21 & 2 & 33 \\
\hline 11 & 26 & 17 & 18 & 34 & 9 \\
\hline 25 & 12 & 19 & 20 & 3 & 28 \\
\hline 7 & 36 & 22 & 13 & 8 & 27 \\
\hline 35 & 4 & 15 & 24 & 29 & 10 \\
\hline 114 & 116 & 112 & 110 & 106 & 108 \\
\hline
\end{tabular}

THEOREM 3. For $n=4 k+2(k=1,2,3, \ldots)$, there exist anti-magic squares of order $n$ both for type - and type + .

Proof. Similar to the construction in previous section (with some small modification), for every integer $k>1$, one can generate a pair of twin anti-magic squares $M_{4 k+2}^{-}$and $M_{4 k+2}^{+}$from $M_{4 k-2}^{-}$or $M_{4 k-2}^{+}$. Especially, $M_{10}^{-}$and $M_{10}^{+}$can be constructed from $Q^{-}$or $Q^{+}$.

As an example, here we write out $M_{10}^{-}$in detail, see Table 8 .

Table 8. $\quad M_{10}^{-}$

\begin{tabular}{|c|c|c|c|c|c|c|c|c|c|}
\hline 3 & 98 & 87 & 13 & 12 & 91 & 8 & 94 & 90 & 1 \\
\hline 97 & 4 & 14 & 88 & 89 & 10 & 93 & 7 & 2 & 92 \\
\hline 86 & 15 & 37 & 64 & 55 & 46 & 62 & 33 & 69 & 32 \\
\hline 16 & 85 & 63 & 38 & 48 & 53 & 34 & 65 & 31 & 70 \\
\hline 17 & 84 & 43 & 58 & 49 & 50 & 66 & 41 & 30 & 71 \\
\hline 83 & 18 & 57 & 44 & 51 & 52 & 35 & 60 & 72 & 29 \\
\hline 82 & 19 & 39 & 68 & 54 & 45 & 59 & 40 & 73 & 28 \\
\hline 20 & 81 & 67 & 36 & 47 & 56 & 42 & 61 & 27 & 74 \\
\hline 11 & 99 & 21 & 79 & 78 & 24 & 25 & 75 & 96 & 6 \\
\hline 100 & 9 & 80 & 22 & 23 & 77 & 76 & 26 & 5 & 95 \\
\hline 515 & 512 & 508 & 510 & 506 & 504 & 500 & 502 & 495 & 498 \\
\hline
\end{tabular}


Combining Theorems 2 and 3, we obtain the answer for the question 2.23 of Abe [1] in the case of all even orders.

Acknowledgment. The author would like to thank the referee for valuable suggestions.

\section{REFERENCES}

[1] G. AвE, Unsolved problems on magic squares, Discrete Math., 127 (1994), 3-13.

DePaRTMENT OF Mathematics

YANGZHOU UNIVERSITY

YANGZHOU, JiANGSU 225002

P. R. CHINA 